\title{
The status of GEO 600
}

H Grote ${ }^{1}$, B Allen ${ }^{2}$, P Aufmuth ${ }^{1}$, C Aulbert $^{2}$, S Babak $^{3}$, R Balasubramanian ${ }^{3}$, B W Barr ${ }^{4}$, S Berukoff ${ }^{2}$, A Bunkowski ${ }^{1}$, G Cagnoli ${ }^{4}$, C A Cantley ${ }^{4}, M$ M Casey $^{4}$, S Chelkowski $^{1}$, D Churches ${ }^{3}$, T Cokelaer ${ }^{3}$, C N Colacino ${ }^{1}$, D R M Crooks ${ }^{4}$, C Cutler $^{2}$, K Danzmann $^{1}$, R Davies ${ }^{3}$, R J Dupuis ${ }^{4}$, E Elliffe ${ }^{4}$, C Fallnich ${ }^{5}$, A Franzen ${ }^{1}$, A Freise ${ }^{1}$, S Goßler ${ }^{1}$, A Grant ${ }^{4}$, S Grunewald ${ }^{2}$, J Harms ${ }^{1}$, G Heinzel $^{1}$, I S Heng ${ }^{1}$, A Hepstonstall ${ }^{4}$, M Heurs ${ }^{1}$, M Hewitson ${ }^{4}$, S Hild ${ }^{1}, \mathbf{J ~ H o u g h}^{4}$, Y Itoh ${ }^{2}$, R Jones ${ }^{4}$, S H Huttner ${ }^{4}$, K Kawabe ${ }^{4}$, C Killow ${ }^{4}$, K Kötter ${ }^{1}$, B Krishnan ${ }^{2}$, V Leonhardt ${ }^{1}$, H Lück ${ }^{1}$, B Machenschalk ${ }^{2}$, M Malec ${ }^{1}, \mathbf{R}$ A Mercer ${ }^{6}$, C Messenger ${ }^{6}$, S Mohanty ${ }^{2}$, K Mossavi $^{1}$, S Mukherjee ${ }^{2}$, P Murray ${ }^{4}$, S Nagano ${ }^{1}$, G P Newton ${ }^{4}$, M A Papa ${ }^{2}$, M Perreur-Lloyd ${ }^{4}$, M Pitkin $^{4}$, M V Plissi ${ }^{4}$, V Quetschke ${ }^{1}$, V Re $^{6}$, S Reid $^{4}$, L Ribichini ${ }^{1}$, D I Robertson ${ }^{4}$, N A Robertson ${ }^{4,7}$, J D Romano ${ }^{3}$, S Rowan ${ }^{4}$, A Rüdiger ${ }^{1}$, B S Sathyaprakash ${ }^{3}$, R Schilling ${ }^{1}$, R Schnabel ${ }^{1}$, B F Schutz ${ }^{2,3}$, F Seifert $^{1}$, A M Sintes ${ }^{8}$, J R Smith ${ }^{1}$, P H Sneddon ${ }^{4}$, K A Strain ${ }^{4}$, I Taylor ${ }^{3}$, R Taylor ${ }^{4}$, A Thüring ${ }^{1}, \mathbf{C}$ Ungarelli $^{6}$, $\mathbf{H}$ Vahlbruch ${ }^{1}, \mathbf{A}$ Vecchio $^{6}, \mathbf{J ~ V e i t c h ~}^{4}, \mathbf{H}$ Ward $^{4}$, U Weiland $^{1}$, H Welling ${ }^{5}$, P Williams ${ }^{2}$, B Willke ${ }^{1}$, W Winkler ${ }^{1}$, G Woan ${ }^{4}$ and I Zawischa ${ }^{5}$

\footnotetext{
${ }^{1}$ Max-Planck-Institut für Gravitationsphysik (Albert-Einstein-Institut) und Universität Hannover, Callinstr. 38, D-30167 Hannover, Germany

${ }^{2}$ Max-Planck-Institut für Gravitationsphysik (Albert-Einstein-Institut), Golm Am Mühlenberg 1, 14476 Golm, Germany

${ }^{3}$ Department of Physics and Astronomy, Cardiff University, PO Box 913, Cardiff CF2 3YB, UK

${ }^{4}$ Department of Physics \& Astronomy, University of Glasgow, Glasgow G12 8QQ, UK

${ }^{5}$ Laser Zentrum Hannover e. V., Hollerithallee 8, 30419 Hannover, Germany

${ }^{6}$ School of Physics and Astronomy, The University of Birmingham, Edgbaston, Birmingham B15 2TT, UK

${ }^{7}$ Edward L Ginzton Laboratory, Stanford University, Stanford, CA 94305-4088, USA

${ }^{8}$ Departament de Fisica, Universitat de les Illes Balears, E-07122 Palma de Mallorca, Spain

E-mail: hartmut.grote@aei.mpg.de
}

Received 27 October 2004

Published 21 April 2005

Online at stacks.iop.org/CQG/22/S193

\section{Abstract}

Since December 2003, the gravitational-wave detector GEO 600 has routinely operated in the dual recycled mode, using a lock acquisition scheme based on the detection of optical sideband power at the dark port. With the detector locking very robustly, the current commissioning work is entirely dedicated to sensitivity improvements. We give a brief overview of the GEO 600 detector, 
the implementation of dual recycling, and summarize recent work regarding the increase in the detector sensitivity.

PACS numbers: $04.80 . \mathrm{Nn}$, 07.60.Ly, 95.55.Ym

(Some figures in this article are in colour only in the electronic version)

\section{Introduction}

The British-German laser-interferometric gravitational-wave detector GEO 600 is part of the worldwide network of detectors aiming at the direct detection of gravitational waves. GEO 600 is a dual recycled Michelson interferometer with single folded arms within vacuum tubes of $600 \mathrm{~m}$ length. Dual recycling is the combination of power recycling and signal recycling, and it is used to enhance the shot-noise-limited sensitivity of the interferometer [1-3]. Figure 1 shows a schematic of GEO's optical configuration. A broader description of the basic set-up can be found in [4]. In addition to dual recycling, the GEO 600 detector uses monolithic ultimate pendulum stages in conjunction with triple pendulum chains [5]. Electrostatic drives are engaged as high-frequency actuators for the Michelson differential degree of freedom [6]. All of these techniques are planned to be installed in future revisions of other kilometre-scale detectors, such as for example the Advanced LIGO detector.

\section{Implementation of dual recycling}

GEO 600 is the first of the kilometre-scale detectors to use dual recycling, and its successful implementation is an experimental validation of its principles on detectors of this scale. With a signal recycling mirror being present at the dark port (also commonly called the 'antisymmetric port'), the signal sidebands carrying gravitational wave information are resonantly enhanced within the cavity formed by the Michelson interferometer and the signal recycling mirror. The microscopic longitudinal position of the signal recycling mirror (the 'tuning') determines the frequency of maximum signal enhancement and has to be controlled by a feedback loop. However, the implementation of this mirror introduces strong couplings between the three different control signals for the longitudinal degrees of freedom: the power-recycling cavity length, the difference between the Michelson armlengths and the length of the signalrecycling cavity. Figure 2 shows an overview of the longitudinal control scheme for the dual recycled Michelson. The power-recycling lock employs the reflection-locking technique [7] to obtain a signal that represents the difference between the laser frequency and the eigenfrequency of the power-recycling cavity. Phase modulation sidebands (passing MC2 on its first free spectral range) are imposed onto the light by EOM1. The light returning from the power-recycling cavity is sensed by photodetector PDPR and feedback is applied to MC2 length (forcing the laser to follow in frequency) and EOM2 (acting as fast phase corrector up to $30 \mathrm{kHz}$ ), in order to keep the laser light on resonance with the power-recycling cavity.

In order to obtain a control signal for locking of the Michelson interferometer to the dark fringe, frontal phase modulation at a free spectral range of the power-recycling cavity is applied by EOM 3. Due to an intended asymmetry between the two Michelson arms of about $7 \mathrm{~cm}$, a small fraction of the modulation sidebands leak to the dark port where they are used as local oscillators, beating with the gravitational-wave signal sidebands, also present at the dark 


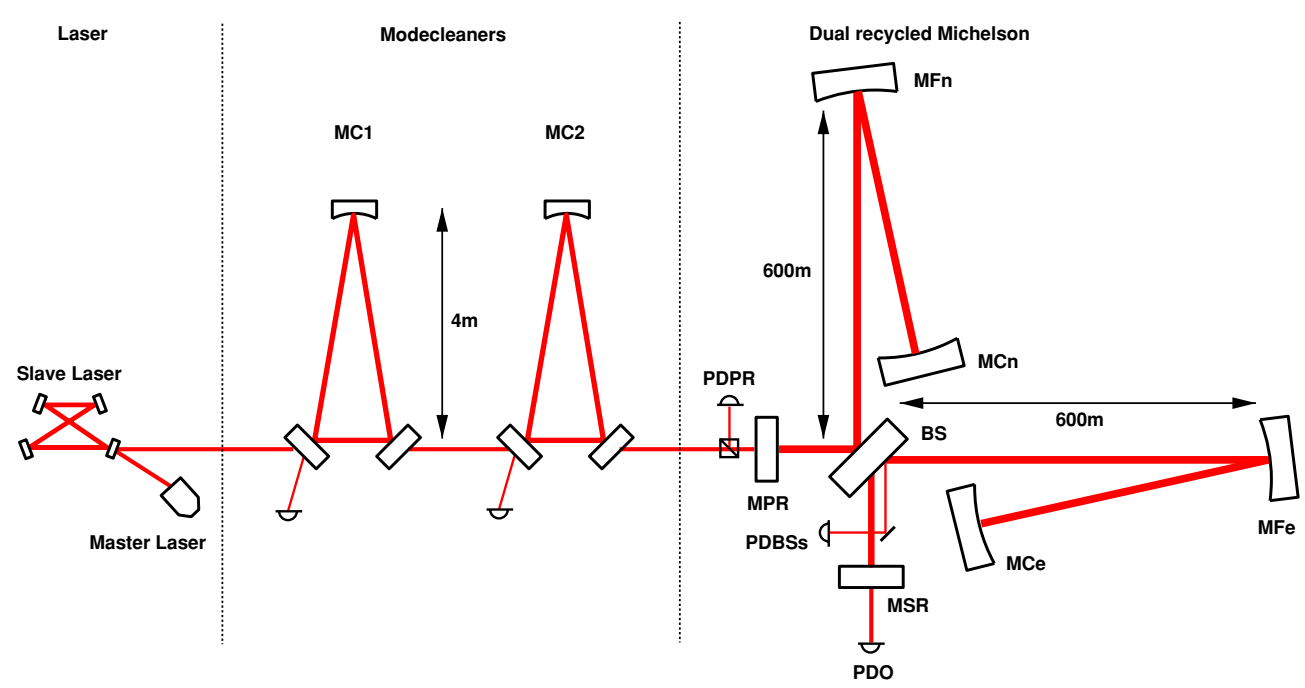

Figure 1. Simplified optical scheme of GEO 600. The two-stage master and slave laser system is followed by two suspended ring-type modecleaners. The main interferometer comprises the Michelson with the folded arms (consisting of beamsplitter BS, and mirrors MCE, MCN, MFE and MFN), together with the power recycling and signal recycling mirrors (MPR and MSR).

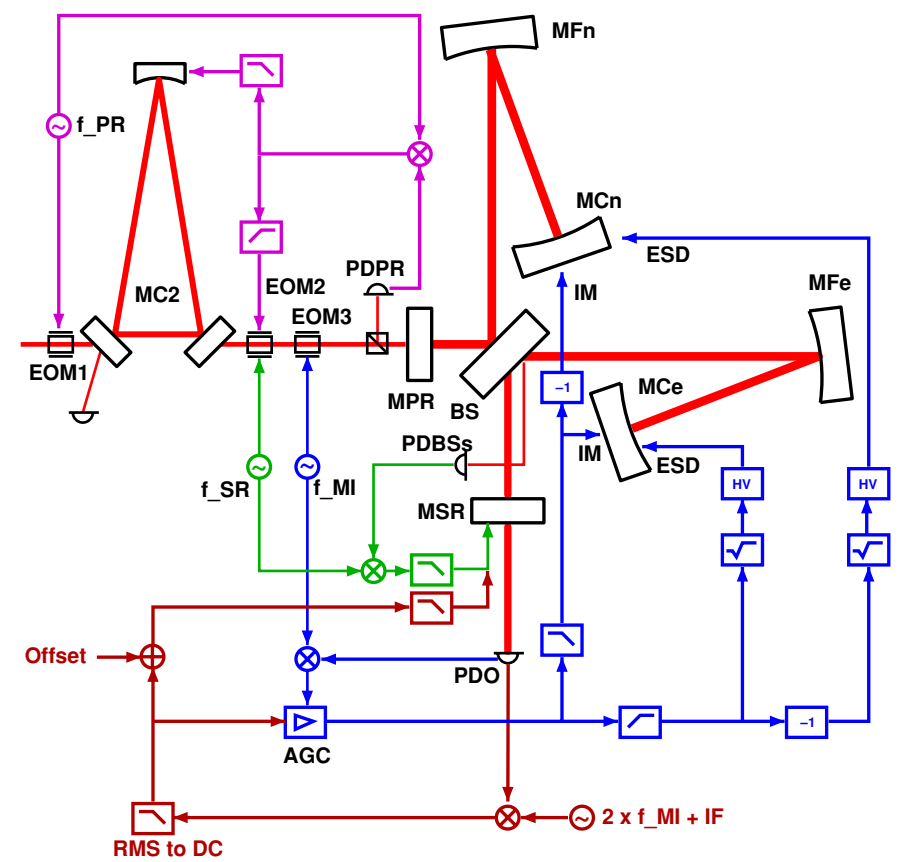

Figure 2. Control scheme of the three longitudinal degrees of freedom for the dual recycled Michelson interferometer. See the text for details.

port. The demodulated signal of detector PDO contains the differential-length information of the Michelson and is fed back to the central Michelson mirrors MCE and MCN. For this servo 
path, GEO 600 uses a split feedback to electrostatic drives (ESD) at the testmass level and coil and magnet actuators at the intermediate mass (IM) level, located penultimate to the testmass. To linearize the force response of the ESDs with respect to a drive voltage, the square root of the drive voltage is processed with analogue electronics, before being applied to high-voltage amplifiers (HV). The square-root circuit is only used for the lock acquisition process, and can be bypassed in the final run mode.

The error signal for the longitudinal control of the signal recycling mirror MSR is obtained by applying a separate phase modulation (by EOM 2) to the light incident on the power recycling cavity. The fraction of the beam reflected off the beamsplitter's anti-reflective-coated side from the east arm is detected by PDBSs and the resulting signal is (after demodulation and filtering) fed back to MSR in the normal operating state via coil and magnet actuators at the mirror level.

Bringing the interferometer to the locked state however, requires a lock acquisition process which takes various signal couplings into account. The locking of the dual recycling configuration can be separated into three phases: the power recycling cavity has to be locked first, in order to have significant light power available at the Michelson interferometer. Then the Michelson can be locked to the dark fringe and finally the signal recycling mirror is locked to its chosen tuning point. Initial experiments with GEO 600 showed that locking this way is possible in principle, but far from being practically usable. While the signal recycling mirror moves longitudinally uncontrolled, the gain of the Michelson control signal (which is proportional to the optical gain of the Michelson) is largely varied, thus making feedback control susceptible to instabilities. The signal recycling locking signal is largely dependent on the deviation of the Michelson from the dark fringe and other parameters, such as for example beam alignment. Due to this fact, a deviation of the Michelson from the dark fringe of the order $1 \mathrm{~nm}$ makes the signal recycling control signal flip sign and hence makes it unusable for feedback control purposes [6]. While $1 \mathrm{~nm}$ is a large deviation from the dark fringe, once the interferometer is in the normal operating condition, it is not so during the acquisition process of the interferometer. Therefore it turned out to be of great benefit, if a signal for the locking of the signal recycling mirror can be obtained which does not critically depend on the deviation of the Michelson from the dark fringe and on other parameters of the interferometer, such as for example, alignment.

A suitable signal for this control step is the power at twice the Michelson Schnuppmodulation frequency, appearing at the dark port photodiode (PDO). This signal (which we call the ' $2 f$ ' signal) is a measure of the Schnupp-sideband power within the signal-recycling cavity. Simulations [8] show that the optical gain of the Michelson (that means the slope of the Michelson error signal with respect to Michelson differential displacement) is roughly proportional to the Schnupp-sideband power for most tunings of the signal recycling mirror (MSR). With the optical gain being mainly determined by the tuning of MSR, the $2 f$-signal can be used for the longitudinal control of MSR. Further, in the implemented locking scheme, the $2 f$-signal is also used to normalize the Michelson control loop gain during the lock acquisition process ('AGC' in figure 2). The $2 f$-signal is obtained by demodulating the signal of output detector PDO at twice the Michelson modulation frequency plus an intermediate frequency IF $=30 \mathrm{kHz}$. Detecting the rms-level of the resulting signal (in the band centred around IF) then yields the power of the $2 f$-signal (independent of any demodulation phase). As the measurement of an optical power is an asymmetric signal, an offset is subtracted to obtain a suitable control signal. This signal is then used for initially locking the signal recycling mirror to an operation point that corresponds to a tuning to a high Fourier frequency. With this scheme, the typical lock acquisition time is drastically reduced from the order of days to minutes. 


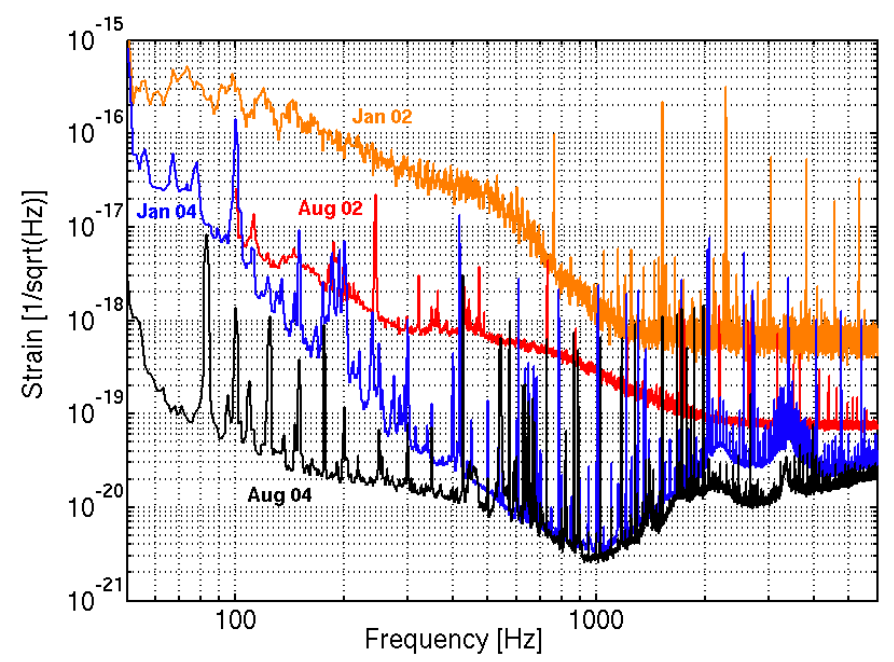

Figure 3. Development of the GEO 600 sensitivity. Compared to the trace of Jan. 2004 ('Jan 04', the typical sensitivity at the end of the S3-science run), the current sensitivity is improved by a factor of approximately 100 at $100 \mathrm{~Hz}$.

If the lock has settled in this state (which includes the switching to higher gain in the Michelson loop and enabling Michelson autoalignment), the signal recycling mirror control can be switched to the nominal sideband-generated signal which has lower noise. Finally, the signal recycling tuning frequency is changed to the chosen operating point while maintaining the locked condition. The change of the tuning frequency is accompanied by the change of five other parameters: the two loop gains and phases for the Michelson and signal recycling mirror lock and the gain for the Michelson alignment loop. This tuning procedure is fully automated (using look-up tables) by the computer control system of GEO 600 [9].

In the final tuned state, we use automatic alignment control for all suspended mirrors of GEO 600 [10], including the signal recycling mirror. For this purpose, the spatial pattern of the beam used for the longitudinal control of MSR is measured with a quadrant-photodiode (denoted by PDBSs in figure 2), yielding differential-wavefront-sensing [11] information of MSR with respect to the east arm of the Michelson. Currently the alignment loops for the power recycling cavity and the Michelson have a larger bandwidth than the signal recycling alignment, such that the alignment signal for MSR is obtained without computing linear combinations from different ports.

\section{Recent and future work}

The whole system has demonstrated its high robustness reaching a duty cycle of about $98 \%$ during 14 days of the S3 science run at the end of 2003 [12]. Since then further improvements of the sensitivity have been achieved. In particular, the sensitivity below $1 \mathrm{kHz}$ was improved by switching the Michelson control from a quadrant-photodiode used as acquisition detector (with a large signal range) to a separate high-power single-element detector, once the stable locked state is established. Further, the output optics used to focus the beam at the dark port of the Michelson has now partly been suspended and placed within vacuum chambers. New electronics with improved gain distribution for the Michelson feedback has been implemented. Noise being introduced from the signal recycling loop has been decreased by reducing the 
loop bandwidth while maintaining stability of the signal recycling mirror control. Reducing the modulation index for the signal recycling loop improved the sensitivity for some frequency ranges above $1.5 \mathrm{kHz}$. This effect is currently under investigation and will require further simulations regarding the distribution of the modulation sidebands within the interferometer. Figure 3 shows a typical sensitivity of August 2004 (lowest trace), compared to previous states. For further improvements, a better understanding of the effect of higher-order spatial beam modes at the dark port is required, as experiments show that the Michelson noise in the region of $1 \mathrm{kHz}$ and above is increased if the output contrast is decreased. (The contrast can be decreased by adjusting the thermal compensation of the radius of curvature of mirror MCE [13].) This is the case despite the fact that the dual recycled GEO 600 has typically a high contrast (carrier light power at dark port divided by light power incident at the beamsplitter) of at least $10^{-5}$.

Future steps will include the rise of the laser power to the final value of $5 \mathrm{~W}$ incident to the power-recycling mirror (currently $1 \mathrm{~W}$ ). Further we plan to increase the power recycling factor from 300 to about 2000. Given the high contrast of GEO 600, it is not clear yet whether we will need to install an output modecleaner.

\section{Acknowledgments}

The authors would like to thank PPARC in the UK, the BMBF and the state of Lower Saxony in Germany.

\section{References}

[1] Meers B J 1988 Phys. Rev. D 38 2317-26

[2] Strain K A and Meers B J 1991 Phys. Rev. Lett. 66 1391-4

[3] Heinzel G et al 1998 Phys. Rev. Lett. 81 5493-6

[4] Willke B et al 2002 Class. Quantum Grav. 19 1377-87

[5] Goßler S, Cagnoli G, Crooks D R M, Lück H, Rowan S, Smith J R, Strain K A, Hough J and Danzmann K 2004 Class. Quantum Grav. 21 922-33

[6] Grote H 2003 PhD Thesis University of Hannover

[7] Drever R W P, Hall J L, Kowalski F V, Hough J, Ford G M, Munley A J and Ward H 1983 Appl. Phys. B 31 97-105

[8] Freise A, Heinzel G, Lück H, Schilling R, Willke B and Danzmann K 2004 Class. Quantum Grav. 21 1067-74

[9] Casey M M, Ward H and Robertson D I 2000 Rev. Sci. Instrum. 71 3910-7

[10] Grote H et al 2004 Class. Quantum Grav. 21 441-50

[11] Morrison E, Meers B J, Robertson D I and Ward H 1994 Appl. Opt. 22 5041-9

[12] Smith J et al 2004 Class. Quantum Grav. 21 1737-45

[13] Lück H et al 2004 Class. Quantum Grav. 21 985-90 